POS PROCEEDINGS

\title{
The DAMPE silicon tungsten tracker
}

\author{
V. Gallo*1, G. Ambrosi², R. Asfandiyarov1 ${ }^{1}$, P. Azzarello1, P. Bernardini ${ }^{3,4}$, \\ B. Bertucci ${ }^{2,5}$, A. Bolognini' ${ }^{2,5}$, F. Cadoux ${ }^{1}$, M. Caprai ${ }^{2}$, I. De Mitri ${ }^{3,4}$, M. Domenjoz ${ }^{1}$, \\ Y. Dong ${ }^{6}$, M. Duranti ${ }^{2,5}$, R. Fan ${ }^{6}$, M. Franco ${ }^{7}$, P. Fusco ${ }^{7,8}$, F. Gargano ${ }^{7}$, K. Gong ${ }^{6}$, \\ D. Guo ${ }^{6}$, C. Husi ${ }^{1}$, M. Ionica ${ }^{2}$, N. Lacalamita ${ }^{7}$, D. La Marra ${ }^{1}$, F. Loparco ${ }^{7,8}$, \\ G. Marsella ${ }^{3,4}$, M.N. Mazziotta ${ }^{7}$, M. Mongelli ${ }^{7}$, A. Nardinocchi ${ }^{2,5}$, L. Nicola ${ }^{1}$,

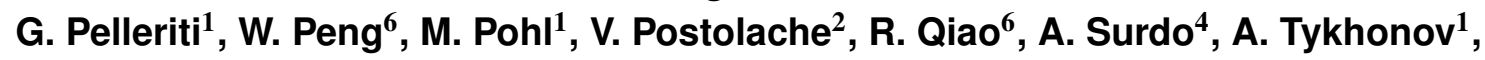



${ }^{1}$ Département de Physique Nucléaire et Corpusculaire, Université de Genève, Geneva, Switzerland

${ }^{2}$ Istituto Nazionale di Fisica Nucleare Sezione di Perugia, Perugia, Italy

${ }^{3}$ Dipartimento di Matematica e Fisica "E. De Giorgi", Università del Salento, Lecce, Italy

${ }^{4}$ Istituto Nazionale di Fisica Nucleare Sezione di Lecce, Lecce, Italy

${ }^{5}$ Dipartimento di Fisica e Geologia, Università di Perugia, Perugia, Italy

${ }^{6}$ Institute of High Energy Physics, Chinese Academy of Sciences, Beijing, China

${ }^{7}$ Istituto Nazionale di Fisica Nucleare Sezione di Bari, Bari, Italy

${ }^{8}$ Dipartimento di Fisica "M.Merlin" dell'Università e del Politecnico di Bari, Bari, Italy

E-mail: valentina.gallo@unige.ch

The DArk Matter Particle Explorer (DAMPE) satellite has been successfully launched on the 17th December 2015. It is a powerful space detector designed for the identification of possible Dark Matter signatures thanks to its capability to detect electrons and photons with an unprecedented energy resolution in an energy range going from few $\mathrm{GeV}$ up to $10 \mathrm{TeV}$. Moreover, the DAMPE satellite will contribute to a better understanding of the propagation mechanisms of high energy cosmic rays measuring the nuclei flux up to $100 \mathrm{TeV}$. DAMPE is composed of four sub-detectors: a plastic strip scintillator, a silicon-tungsten tracker-converter (STK), a BGO imaging calorimeter and a neutron detector. The STK is made of twelve layers of single-sided AC-coupled silicon micro-strip detectors for a total silicon area of about $7 \mathrm{~m}^{2}$. To promote the conversion of incident photons into electron-positron pairs, tungsten foils are inserted into the supporting structure. In this document, a detailed description of the STK construction and its performance on orbit are reported.

The 25th International workshop on vertex detectors

September 26-30, 2016

La Biodola, Isola d'Elba, ITALY

${ }^{*}$ Speaker. 


\section{Introduction}

The DAMPE (DArk Matter Particle Explorer) [1] is an astroparticle physics experiment launched on the 17th December 2015 at 00:12 UTC. It is one of the five scientific missions in the framework of the Strategic Pioneer Program on Space Science of the Chinese Academy of Sciences (CAS).

The DAMPE detector has been designed to detect electrons and positrons in an energy range from few $\mathrm{GeV}$ up to $10 \mathrm{TeV}$ and with a geometrical acceptance of $\sim 0.3 \mathrm{~m}^{2}$ sr. The detection energy range for cosmic rays goes from $10 \mathrm{GeV}$ to above $100 \mathrm{TeV}$. The objectives of the DAMPE experiment are the searches for Dark Matter decay or annihilation signatures, gamma-ray astronomy and cosmic rays flux and composition.

The detector layout is shown in Fig. 1. It is made of the following sub systems: a double layer plastic scintillator strip detector (PSD) used as anti-coincidence for incoming photons and for the measurement of the charge (Z) of incident particles; a silicon-tungsten tracker-converter (STK) which is the main subject of this report and will be described in detail in the following sections; a bismuth germanium oxide imaging calorimeter (BGO) of about 31 radiation lengths for precise energy measurements and for electron/photon identification; a neutron detector (NUD) to improve the electron/proton separation power.

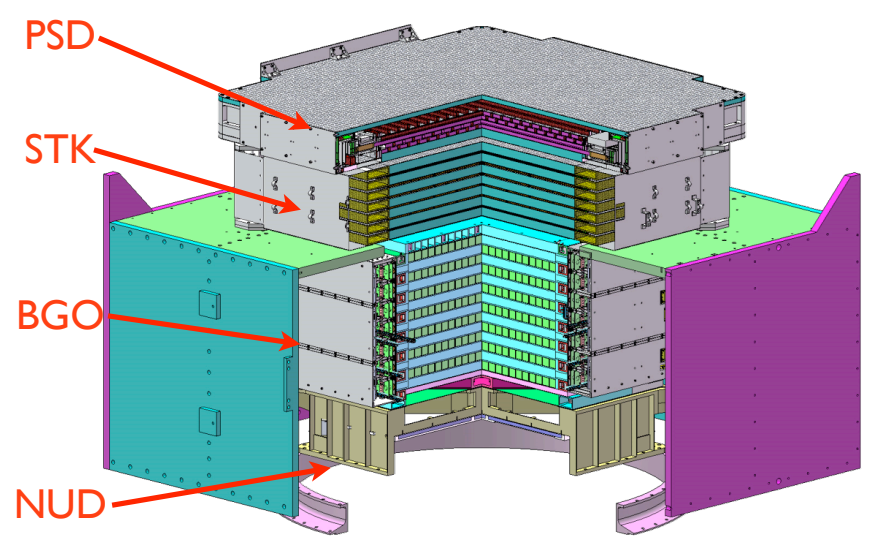

Figure 1: Layout of the DAMPE detector.

After an intense period of prototyping, tests and construction, the STK was completed in April 2015. In the following, after a brief description of the STK, we report on its construction and performance on orbit.

\section{The silicon tungsten tracker}

The main objectives of STK $[2,3,4]$ are: the reconstruction of the trajectories of charged particles, the absolute ion charge identification and the photon conversion in electron-positron pairs. The tracker profits of the well established silicon micro-strip detector technology already used in space experiments such as PAMELA [5], AGILE [6], Fermi [7] and AMS-02 [8]. 
The STK is made of six tracking layers each of them made of two single sided silicon detector layers to measure the two orthogonal views perpendicular to the particle direction. The total silicon area is of $\sim 7 \mathrm{~m}^{2}$, comparable with the AMS-02 tracker surface. Three $1 \mathrm{~mm}$ thick tungsten layers are placed before the second, the third and the fourth tracking point for the photon conversion. In this configuration, the first point provides the entrance position of the charged particle, while in case of photon it behaves as veto. The last three points, without tungsten, allow to reconstruct the tracks of the electron-positron pairs generated from the photon conversion. The multiple scattering effect is minimised using multiple and thin tungsten layers and is found to be negligible for particles with energy above $5 \mathrm{GeV}\left(\theta_{0}=0.08^{\circ}\right.$ for $1 \mathrm{~mm}$ tungsten and $5 \mathrm{GeV}$ particles). The STK total radiation length is of $\sim 1 \mathrm{X}_{0}$.

\subsection{Detector modules}

The STK is made of 768 single sided AC-coupled silicon micro-strip detectors manufactured by Hamamatsu Photonics [9]. The sensors have the same geometry as the ones used by AGILE [10], but with different thickness, bulk resistivity and backplane metallisation. The detector size is of $95 \times 95 \times 0.32 \mathrm{~mm}^{3}$ and each sensor is segmented in 768 strips. The strips are $48 \mu \mathrm{m}$ wide and $93.196 \mathrm{~mm}$ long with a pitch of $121 \mu \mathrm{m}$. The bulk resistivity is $>7 \mathrm{k} \Omega \mathrm{cm}$ with a full depletion voltage of $55 \mathrm{~V}$ maximum. The average single sensor leakage current is of $116 \mathrm{nA}$ at $150 \mathrm{~V}$ well below the specification of $900 \mathrm{nA}$.

Four silicon sensors are assembled together to form one module, named ladder, as shown in Fig 2. The sensors are glued on the $380 \mathrm{~mm}$ long flex extension of the tracker front-end hybrid

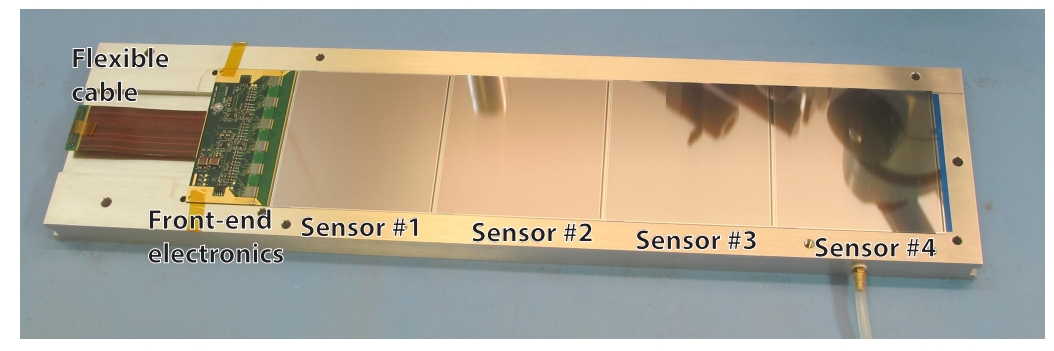

Figure 2: STK ladder made of four single sided AC-coupled silicon micro-strip sensors. The front end electronics is placed at the edge of the TFH which serves also as mechanical support for the silicon sensors. The flexible cable is directly connected to the DAQ board.

board (TFH). The bias voltage is brought by the flex part to the backplane of the silicon sensors. The signal shaping and amplification is done by six ASICS chips VA140 mounted on the TFH and produced by IDEAS [11]. The chip design is based on the one used in AMS-02 [12]. Each VA140 reads 64 channels, for a total of 384 channels per ladder. Hence, only every other strip is readout to limit the number of readout channels while keeping a good performance in terms of spatial resolution. As reported in $[3,13]$, the spatial resolution is better than $50 \mu \mathrm{m}$ for particle incidence angles lower than $40^{\circ}$.

Once the ladder is assembled, the position of each silicon sensor is measured with a metrology machine. The alignment error for the 192 ladders composing the STK is less than $4 \mu \mathrm{m}$, as show from the left side of Fig. 3, and well below the specification of $40 \mu \mathrm{m}$. The ladders operate with a 
bias voltage of $80 \mathrm{~V}$ and with an average leakage current of $330 \mathrm{nA}$ (see right side of Fig. 3). The
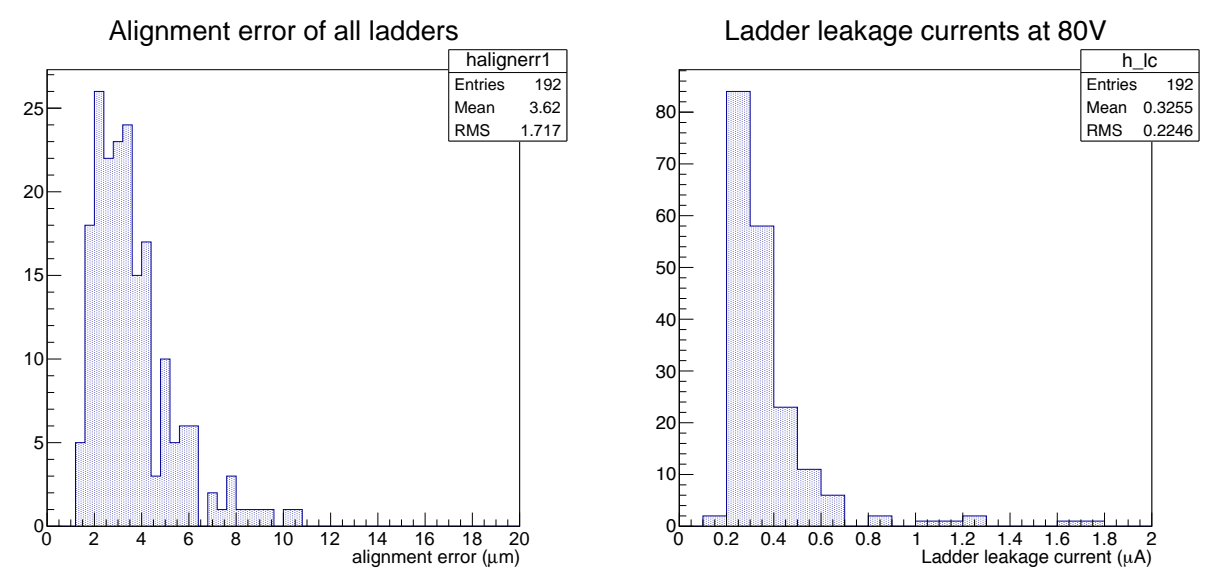

Figure 3: (Left) Alignment error of the 192 ladders of STK. The average alignment error is of $4 \mu \mathrm{m}$, well below the requirement of $40 \mu \mathrm{m}$. (Right) Ladder leakage current for the 192 ladders installed on STK at an operation voltage of $80 \mathrm{~V}$. The average leakage current is of $330 \mathrm{nA}$.

distribution shows the ladders leakage current measured after their installation on the STK. The current of $330 \mathrm{nA}$ confirms the high quality maintained during the STK assembly.

\subsection{STK tracker plane and assembly}

The STK mechanical structure is made of 7 supporting trays of aluminium honeycomb layers sandwiched between two Carbon Fibre Reinforced Polymer (CFRP) face sheets of $0.6 \mathrm{~mm}$ thick for the planes without tungsten, and $1 \mathrm{~mm}$ thick for the others. The second, third and fourth planes are equipped with $1 \mathrm{~mm}$ thick tungsten plates glued into the CFRP sheet inside the tray. The overall structure is light but rigid in order to withstand the vibrations and accelerations due to the rocket launch. The trays have been produced by Composite Design Sàrl [14]. The alignment of each tungsten plate with respect to the 4 corners of the tray has been checked with a X ray scan at CERN.

The two sides of the five inner trays are equipped with 16 ladders each, while for the top and bottom planes only one face is equipped with the silicon modules. The ladders are glued to the plane using an alignment and transfer jig placed on a precision rotating stand, shown in the left side of Fig. 4 and designed to glue two ladders at a time. After the plane is assembled, the position on the tray and the flatness of each silicon sensor is measured with a metrology machine as shown in the right side of Fig. 4. The sensors are found to be flat to within $\sim 100 \mu \mathrm{m}$.

The planes are piled up together to form the full tracker, as shown in Fig 5. The silicon ladders on the bottom surface of one tray are placed orthogonally with respect to the ones of the top surface of the below tray in order to measure the X-Y coordinates of the incident particles. The inter-distance between two consecutive silicon layers is of $\sim 3 \mathrm{~mm}$. Hence, the STK provides a total of 12 measurement points, 6 in $\mathrm{X}$ and 6 in $\mathrm{Y}$.

The Tracker Readout Boards (TRB) and the aluminum radiators have been mounted on the side of STK, as shown in the exploded view of Fig. 6. The STK uses a passive cooling system made 

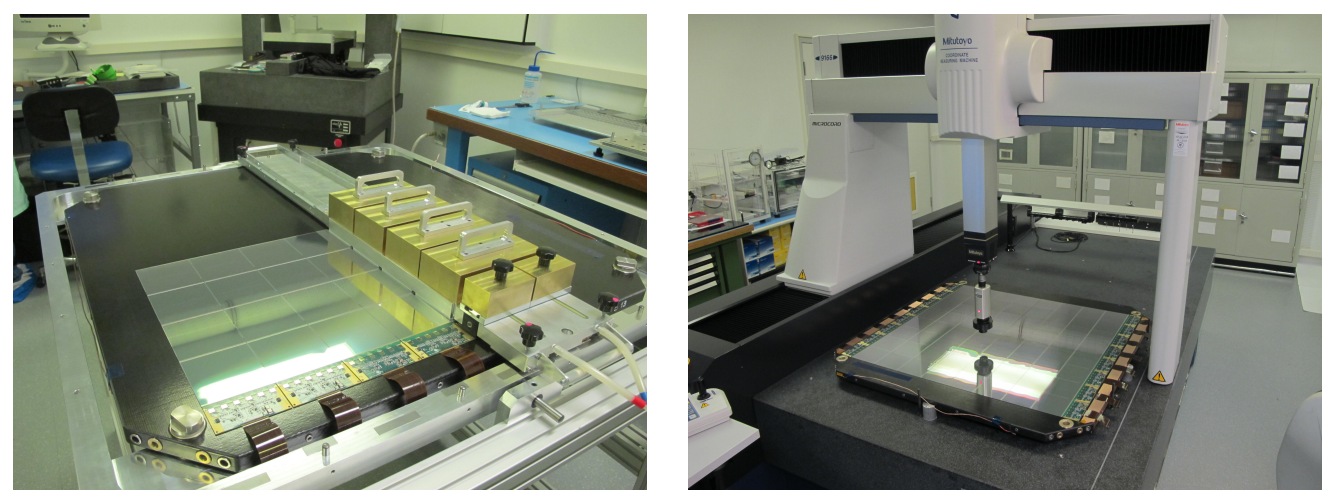

Figure 4: (Left) Ladders gluing on one support tray. The precision jig allows the gluing of two ladders at a time. The tray is fixed to a rotation stand to assemble the ladders on both sides. (Right) Metrology measurement of the position of the sensors of a fully equipped STK plane.



Figure 5: STK before the assembly of the last tray. The 16 ladders belonging to one silicon layer are visible together with the copper bands mounted on the side of the supporting trays and used for the heat transfer.

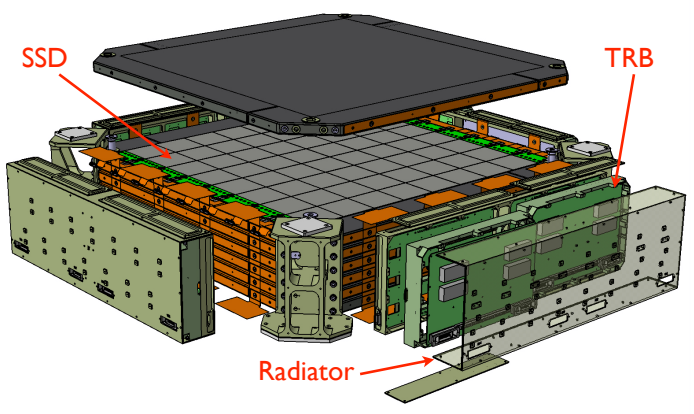

Figure 6: Exploded view of STK. The TRBs are mounted on the fours sides of STK and covered with the radiators. 
of Pyrolytic graphite sheets (PGS) connecting the TFH copper vias to the horizontal copper bands installed on the trays edges. To thermally connect the trays together, copper straps are transversally screwed and glued along the trays. The straps are then connected to the radiators with thermal grease.

\section{STK Performance}

The STK has a total of 73728 channels that have been constantly monitored during the assembly phase. In Fig. 7, the noise distributions for all the STK channels on the ground and on orbit are shown. Two noise thresholds are defined, for channels with noise above 5 or 10 ADC counts.



Figure 7: Noise distribution for the 73728 STK channels. The number of noisy channels is found to be stable before and after the launch. Due to the colder operation temperature on orbit the bulk of the distribution is slightly lower with respect to the one on ground and it is $\sim 2.8$ ADC counts.

As a result of rigorous quality control during all the steps of the assembly, the STK detector has less than $0.4 \%$ of noisy channels before the launch of the satellite and for the lower threshold of 5 ADC counts. This amount of noisy channels is slightly less $(\sim 0.3 \%)$ on orbit due to the colder operation temperature. For the same reason, the bulk of the noise on orbit is $\sim 2.8$ ADC counts and it is lower with respect to ground. The number of channels above the higher noise threshold of 10 ADC counts is stable after the launch and is of $\sim 0.13 \%$.

\subsection{Temperature variation}

The noise of the STK channels depends from the operation temperatures of the apparatus. In order to monitor the ladder temperature two DS18S20 temperature sensors [15] are mounted on each TFH. Temperature sensors are also installed on the STK radiators. The average measured temperature for the radiators and for the ladders as a function of time is shown in Fig. 8. The correlation between those two plots shows how the ladders are well connected to the cooling system. The ladders temperature is constantly $10^{\circ} \mathrm{C}$ higher than the temperature of the radiators. The day 

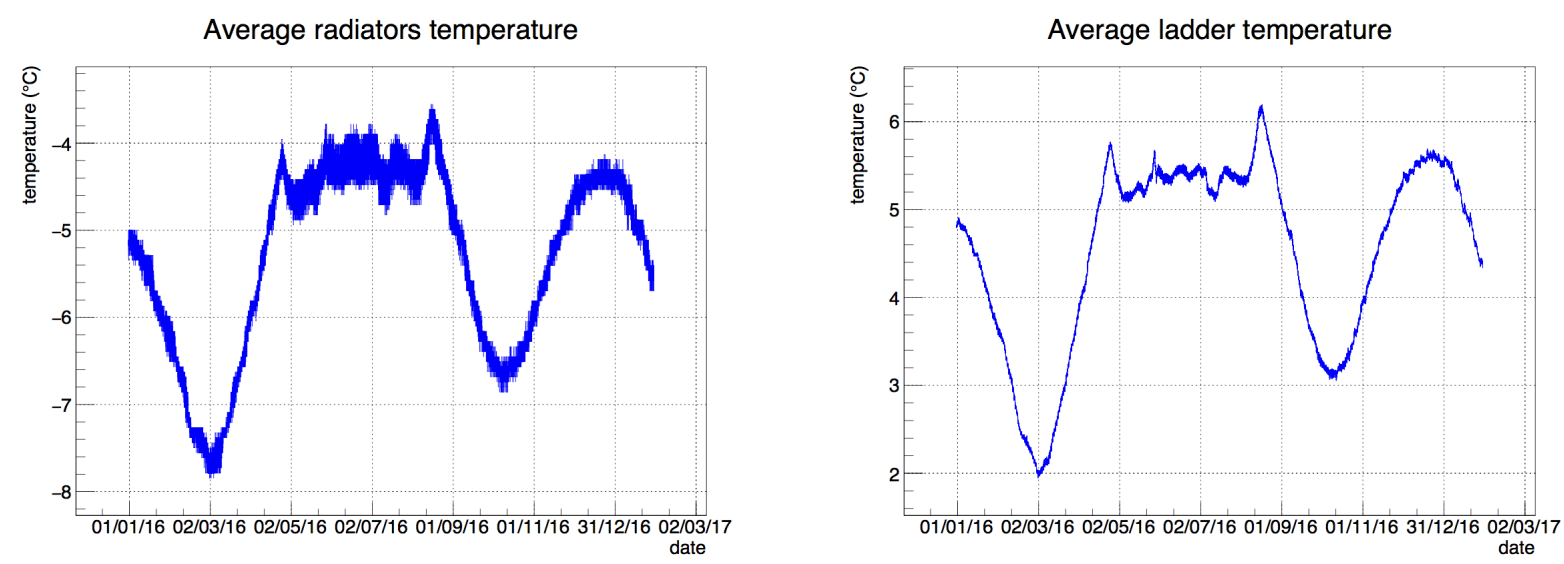

Figure 8: (Left) Average temperature measured on the STK radiators as a function of time. (Right) Average ladders temperature as a function of time. The heat transfer system is properly working, the radiator temperature is constantly $10^{\circ} \mathrm{C}$ lower than the one measured on the ladders.

by day variation is about $0.1^{\circ} \mathrm{C}$ and the maximum monthly variation is of $\sim 2.4^{\circ} \mathrm{C}$. In Fig. 9, the STK channels average noise value is shown as a function of the average ladder temperature. As

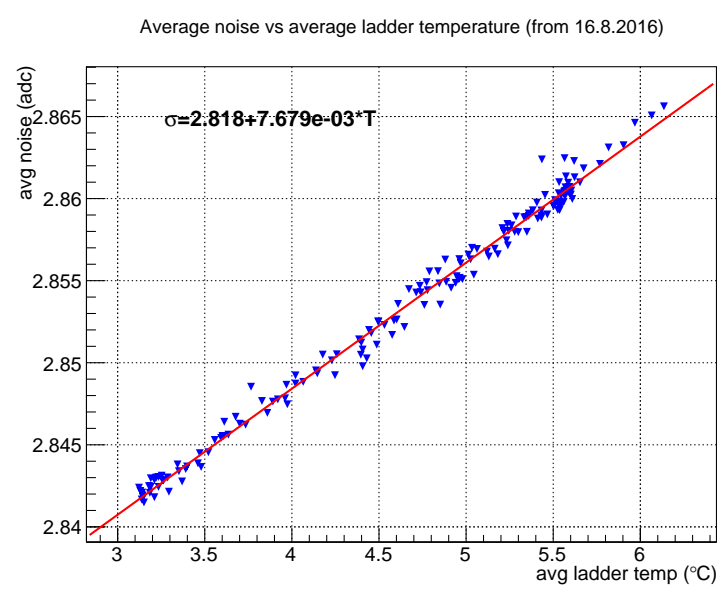

Figure 9: Average noise of the STK channels as a function of average ladder temperature from August 2016 to January 2017. As expected the noise is correlated with the temperature change. The noise variation is found to be of $\sim 0.015 \mathrm{ADC}$ count per $2^{\circ} \mathrm{C}$.

expected, the temperature change induces a subsequent modification of the channel noise. Nevertheless, this variation is very small, and it is found to be $\sim 0.015$ ADC count per $2^{\circ} \mathrm{C}$. This tiny change does not have an impact on the output signal which has a precision of the order of the ADC count. The STK noise is therefore very stable with respect to the temperature variations.

\section{STK readout}

The core of the STK data acquisition is the Tracker Readout Boards (TRB) [16]. The STK is 
equipped of 8 TRBs that are mechanically fixed on the sides of the STK supporting structure, as shown in Fig. 6. Each TRB reads out 24 ladders, for a total of 9216 channels, and is made of three boards. The first one is the ADC board which performs the signal analog to digital conversion. The analog signal is provided by the TFHs that are directly connected to the ADC boards via the flexible cable shown in Fig. 2. The 6 chips of the TFH are divided in two groups of three chips each. The chips from the same group are read in sequence, for a total of 192 clock signals and the two groups are readout in parallel. Each group has its own amplification circuit whose analog outputs are transferred to the ADC board. The second board of the TRB is the FPGA board. It is equipped with two FPGAs dedicated to the communication with the DAMPE DAQ system and the generation of the control signals for the ladder readout and the signal digitalisation. Finally, the Power board which provides the necessary voltages for the front-end electronics, the TRB circuit and the silicon bias voltages. The data size reduction is achieved using a zero-suppression and cluster finding algorithm programmed in the FPGA [17]. The TRB system has been designed and produced by IHEP, Beijing.

\subsection{On orbit VA calibration}

As shown in the previous section, the STK has a total of 1152 VA140 chips dedicated to the signal shaping and amplification. Due to the different response in gain of each chip, a calibration is necessary in order to have an accurate signal analysis. The calibration has been performed using two months of proton events taken on orbit. On the left side of Fig 10, the ADC signal response for six chips belonging to the same ladder is shown as an example. Each distribution has been
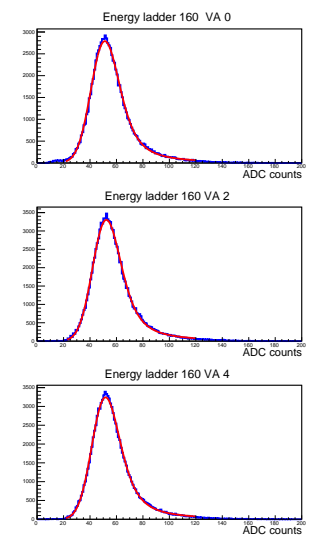
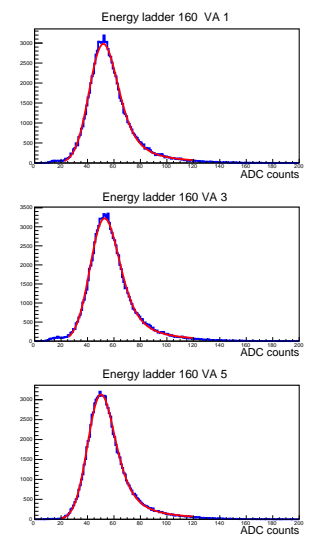



Figure 10: (Left) Example of the signal distribution (blue line) of proton signals on 6 VA140 chips of one ladder. Each distribution is fitted with a Landau convoluted with a Gaussian function, shown in red. (Right) Maximum fit value before (blue distribution) and after the chip gain correction (red distribution).

fitted with a Landau convoluted with a Gaussian function. The maximum value from the fit gives the optimal chip response. As shown on the right side of Fig 10, the wide distribution (in blue) of the chip signal response before the correction is applied extends from 44 up to 60 ADC counts. After the calibration procedure is applied, the signal response turns out to be more uniform with a maximum difference among the chips of few ADC counts. 


\section{Conclusion}

The silicon-tungsten tracker converter (STK) of the DAMPE mission is based on the robust technology of single-sided micro-strip sensors with analog readout. The flight model has been assembled from January to April 2015, then shipped to China where it has passed all the acceptance tests. The STK was integrated in the DAMPE satellite that has been successfully launched on 17th December 2015.

After almost one year from the launch, the STK keeps an excellent quality, in terms of number of noisy channels and temperature stability. An on orbit VA140 response calibration has been performed.

Thanks to the great quality of the STK, this detector is expected to play a crucial role in the gamma-ray detection, nuclear charge particle identification and track reconstruction.

\section{Acknowledgment}

The authors wish to express their gratitude to M. Prest and E. Vallazza of the AGILE Silicon Tracker collaboration for fruitful discussions on tracker design and for kindly allowing us to use the AGILE silicon sensor geometry. The generosity of CERN for providing beam time allocation and technical assistance at the PS and SPS beam lines, as well as general logistical support is acknowledged. This work is supported by the Chinese Academy of Sciences, the Swiss National Science Foundation and INFN, Italy.

\section{References}

[1] Chang, J., Chinese Journal of Space Science, (2014) 34 (5) 550-557

[2] X. Wu et al., PoS(ICRC2015)1192.

[3] P. Azzarello et al., Nuclear Instruments and Methods in Physics Research A 831 (2016) 378-384

[4] Chang, J. et al. [DAMPE collaboration], Astroparticle Physics, to be submitted (2017)

[5] W. Menn et al., Advances in Space Research, 51 (2013) 209-218.

[6] M. Tavani et al, Nuclear Instruments and Methods in Physics Research A 588 (2008) 52-62

[7] W.B. Atwood, et al., The Astrophysical Journal 697 (2) (2009) 1071.

[8] M. Aguilar et al., Phys. Rep. 366 (2002) 331.

[9] http://www.hamamatsu.com/us/en/index.html

[10] G. Barbiellini et al, Nuclear Instruments and Methods in Physics Research A 490 (2002) 146-158

[11] Integrated Detector Electronics AS, http://www.ideas.no

[12] G. Ambrosi et al., Nuclear Instruments and Methods in Physics Research A 435 (1999) 215

[13] V. Gallo et al., PoS(ICRC2015)1199.

[14] http://www.compositedesign.ch

[15] https://datasheets.maximintegrated.com/en/ds/DS18S20.pdf

[16] Fei Zhang et al., arXiv:1606.05080

[17] Dong Yi-Fan et al., Chinese Physics C Vol. 39, No. 11 (2015) 116202 